\title{
Cataract Surgery in a Patient with Amyotrophic Lateral Sclerosis: A Case Report
}

\author{
Maryo Cenk Kohen Raciha Beril Kucumen \\ Department of Ophthalmology, Yeditepe University, Istanbul, Turkey
}

\section{Key Words}

Cataract $\cdot$ Surgery $\cdot$ Amyotrophic lateral sclerosis

\begin{abstract}
We report a cataract operation with complications in a patient with amyotrophic lateral sclerosis (ALS). The patient had a grade 4 mature brown cataract. Phacoemulsification with intraocular lens (IOL) implantation was planned; however, due to unexpected complications occurring during surgery, the operating technique was revised to an intracapsular cataract extraction. A very high vitreous pressure was found and therefore scleral fixating IOL was not implanted after anterior vitrectomy because of the possibility of choroidal effusion. The postoperative visual acuity improved sufficiently for the patient to communicate. Visual communication is of vital importance for an ALS patient and his caregivers. Therefore, surgery may be advisable in patients at a terminal stage with an advanced cataract, even if their general health condition may not seem appropriate for such an operation. Nevertheless, the intra- and postoperative course of the surgery may show unexpected complications and the surgeon should be prepared for such conditions.
\end{abstract}

\section{Introduction}

Amyotrophic lateral sclerosis (ALS) is caused by the loss of motor neurons in the central nervous system, the spine and the brain stem. It occurs mainly in over 50-yearolds and is the most common and severe of motor neuron diseases. The incidence is around 1-2.5 per 100,000. The pathological mechanism killing motor neurons is complex and not well understood. With the loss of motor neurons, the muscles begin to cease functioning and atrophy sets in. Weakness in the muscles may begin in the hands, legs, mouth, pharynx or tongue and then spreads progressively. However, the mental functions and the memory are not harmed by the disease. The spread of the disease may also include the muscles in the bulbar area, and it may impair speech and swallowing. In the 
advanced stages, respiratory failure becomes a possibility with mechanical ventilatory support prolonging survival. The extraocular muscles are rarely affected at the terminal stage. Therefore, visual ability together with eyelid movements may become the only way of communication for patients who have ALS. As their life span lengthens, keeping this communication channel working may become crucially important both for the caretakers and the patient [1].

\section{Case Report}

A 53-year-old, male ALS patient was referred to our clinic with bilateral visual loss. The ALS disease had been diagnosed 10 years ago. The medical history included a bowel cancer operation in 2001, and a tracheostomy and mechanical ventilation following respiratory failure in 2004 . The patient also suffered from aggressive pemphigus vulgaris for which he was under steroid treatment since 2005 . The ophthalmological examination was done while the patient was lying in his bed. His only way of communication was opening and closing his eyelids; in order to answer 'yes' he would close his eyelids once and to answer 'no' he would widen his eyelids as much as he could. He was also able to form simple sentences by signaling yes or no to the letters shown from an alphabet chart prepared for him. The refractive status of the patient could not be measured with a portable auto refractometer (Retinomax K-Plus 2; Righton, Tokyo Japan).

The uncorrected visual acuity was assessed by counting fingers from $10 \mathrm{~cm}$ for both eyes; neither pinhole nor random high + or - glasses increased the vision. His previous spectacles were -5.0 diopters (D) for both eyes. A mature brunescent cataract was diagnosed by slit-lamp examination at the bed side using a portable slit-lamp (SL-15; Kowa, Tokyo, Japan). Ocular surface and corneas were normal in both eyes and the intraocular pressure was also normal (tono-pen; Medtronic Saval, Jacksonville, Fla., USA). Indirect and direct ophthalmoscopy was not successful due to the density of the cataract.

The axial length was 26.44 and $26.30 \mathrm{~mm}$ in the right and left eye, respectively. The keratometric measurements were $40.0 / 40.75 \mathrm{D}$ in the right eye and $39.87 / 41.00 \mathrm{D}$ in the left eye. The visual failure appeared to be due to the development of a bilateral cataract. Following the biometrical measurements and a consultation with the Department of Anesthesiology, the patient was scheduled for cataract surgery under local anesthesia.

On the day of surgery a preliminary problem arose as the patient had to be transported from his bed to the operating table. The patient's body and limbs showed extreme fragility due to severe osteoporosis, muscle atrophy and pemphigus. For this reason the patient could not be transported. Consequently, it was decided to perform the operation on the patient's own bed supported by numerous cushions (fig. 1). In order to provide the sterility of an operating theatre, the whole bed, including the wheels, and the patient were covered with sterile sheets. To adequately see the anterior segment through the operation microscope, the surgeon had to bend over the patient, which proved to be a rather uncomfortable task.

The operation started as a routine phacoemulsification under sub-Tenon's anesthesia with a 3.0-mm slit knife incision followed by capsule staining with $1 \%$ trypan blue. A continuous curvilinear capsulorhexis of $5.5 \mathrm{~mm}$ diameter was performed, followed by hydrodissection. At the beginning of the phacoemulsification, excessive mobility of the lens was noticed. There was significant zonular dehiscence and absence in the nasal quadrants of more than 180 degrees and the anterior chamber deepened in these quadrants as well. Due to the threat of a lens drop, the procedure was changed into an intracapsular lens extraction with 160-degree corneal incision. The lens including its capsule was expressed easily followed by a spontaneous vitreous prolapsus with intact anterior surface. Following anterior vitrectomy the cornea was sutured with 10.0 nylon single sutures. The eye was left aphakic because of excessive vitreous prolapsus; the possibility of choroidal effusion and/or suprachoroidal hemorrhage deterred us from the time-consuming scleral-fixated intraocular lens implantation (fig. $2 \mathrm{a}-\mathrm{g}$ ).

The patient's visual acuity was $20 / 400$ the next day with $+7.00 \mathrm{D}$ glasses. However, on the third postoperative day an epithelial bulla appeared on the central cornea with a simultaneous systemic exacerbation of the pemphigus. On the fourth day, the corneal bulla erupted and turned into an epithelial defect which was infected secondarily in the following days. Despite treatment with $0.5 \%$ 
moxifloxacin eye drops and tobramycin ointment, the keratitis did not heal. The patient also had onychomycosis in his hands and feet for a long time and since he was under immunosuppressive therapy, a fungal keratitis was considered. As a result the topical steroids were immediately tapered and antifungal topical therapy (natamycin 5\% ointment) was initiated. After 21 days of therapy the keratitis healed leaving a light scar. The patient was satisfied with his visual capacity and expressed a wish that his second eye be operated upon, too. Sadly, 2 months later he died during hospitalization due to complications arising from a hip fracture.

\section{Discussion}

The unusual nature of this cataract surgery, which was originally planned as phacoemulsification with intraocular lens implantation in a patient with ALS, leaves us with a number of points worthy of attention. First of all, positioning the patient for surgery was a problem that had to be resolved. It is obvious that various systemic conditions may limit the ideal positioning of the patient and even the surgeon during cataract surgery as reported by Muthialu et al. [2]. In our case we overcame this obstacle by modifying the patient's bed into a sterile operating table.

The second problem was significant zonular dehiscence and threat of drop, both preoperatively and in the first stages of the operation. As we performed the corneal incisions and capsulorhexis, we did not observe any alarming signs of these complications. Our patient had neither a history of trauma nor glaucoma. He had only moderate axial myopia, and was relatively young; therefore subtle lens subluxation with impending drop was an unexpected complication for us. Furthermore, the excessive positive vitreous pressure after the expression of the cataract was also a continuation of these intraoperative complications.

ALS is a neurodegenerative disorder of undetermined etiology that primarily affects the motor neurons in the motor cortex, brain stem and spinal cord. It is progressive and most patients eventually end up with respiratory failure. The first detailed description was by Jean Martin Charcot in 1869 in which he discussed the clinical and pathological characteristics of 'la sclerose laterale amyotrophique', a disorder that both affected the upper motor neuron and the lower motor neuron [3].

Ocular motility is not typically affected by ALS and the only ophthalmologically related article about ocular pathology found in ALS patients was concerned with ophthalmomyiasis-like pigmentary retinopathy, this being mentioned by Kokubo et al. in a letter to the editor [4]. They argue that a type of pigmentary retinopathy commonly seen in Guam and the Kii peninsula of Japan, the latter also being a part of the world with a higher incidence of ALS $\backslash$ PDC (ALS and parkinsonism-dementia complex), is somehow related.

Cataracts may be common among ALS patients due to high usage of corticosteroids and other cataract-inducing drugs. However, cataract surgery is performed rarely due to low life expectancy and other illnesses which require more attention, with the obvious result that there is a scarcity of literature about cataract surgery in ALS patients. In our case it was not possible to create a direct link between ALS disease and lens subluxation, but the unexpected extreme frailty and the absence of zonules may be a consequence of ALS, and might, in fact, be considered a symptom of the disease. This may be an important issue to keep in mind when attempting cataract surgery in a patient with ALS. 
The only published paper on this subject is by Kowalik et al. [5]. In this case report, the cataract operation on the first eye was complicated by endophthalmitis and required vitrectomy. Conversely, the cataract operation on the second eye was uneventful. However, this paper is more focused on the anesthesia of the patient.

Pemphigus vulgaris is an immune-mediated vesiculobullous disease of the skin and mucous membranes [6,7]. Even though our patient had shown no signs of ocular pemphigus preoperatively, the postoperative bullae formation was probably due to the exacerbation of the disease. This complication after cataract surgery has not been described before. However, we believe that because of high usage of systemic corticosteroids and immunosuppressant drugs the corneal epithelial defect did not heal and resulted in a fungal keratitis.

Cataract surgery in severely disabling and debilitating diseases such as in ALS should be considered if vision is the only means of communication for the patient. We think that in the event of loss of communication, surgery both helps the caregiver and the patient with morale and well being. It may be important to remember that these surgeries can be complicated and should be performed by skilled and experienced surgeons. In addition, because of the general gravity of the health condition of an ALS patient, the most unexpected postoperative complications may also occur such as corneal epithelial bullae followed by fungal keratitis, as has happened in our case.

\section{Disclosure Statement}

None of the authors has a financial or proprietary interest in any material or method mentioned. 


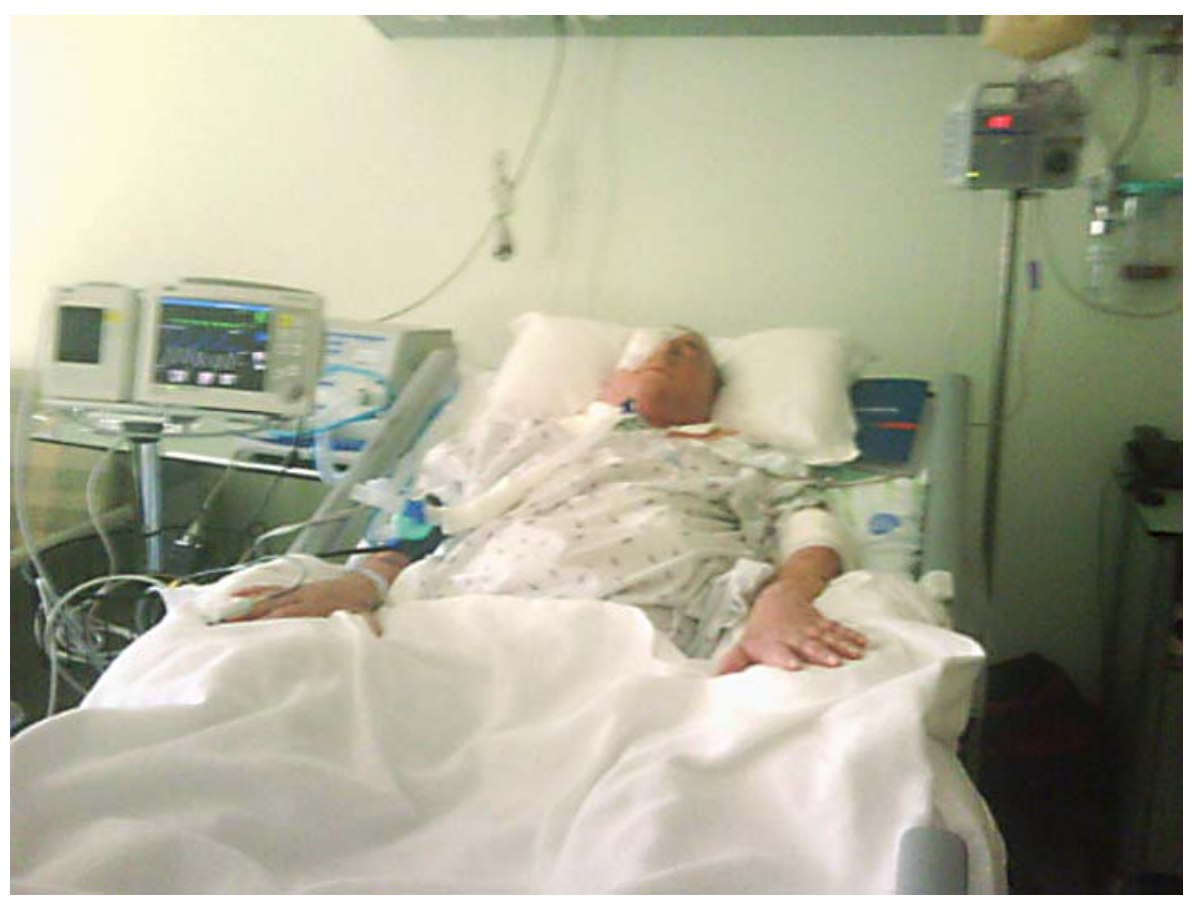

Fig. 1. Patient's appearance 1 day after surgery (with permission of the patient's legal guardian). 


\begin{tabular}{|c|c|c|c|}
\hline $\begin{array}{l}\text { Case Reports in } \\
\text { ohthalmoloov }\end{array}$ & $\begin{array}{l}\text { Case Rep Ophthalmol 2011;2:198-204 } \\
\text { DOI: } 10.1159 / 000329832\end{array}$ & $\begin{array}{l}\text { Published online: } \\
\text { June } 28,2011\end{array}$ & $\begin{array}{l}\text { () } 2011 \text { S. Karger AG, Basel } \\
\text { ISSN } 1663-2699 \\
\text { www.karger.com/cop }\end{array}$ \\
\hline
\end{tabular}
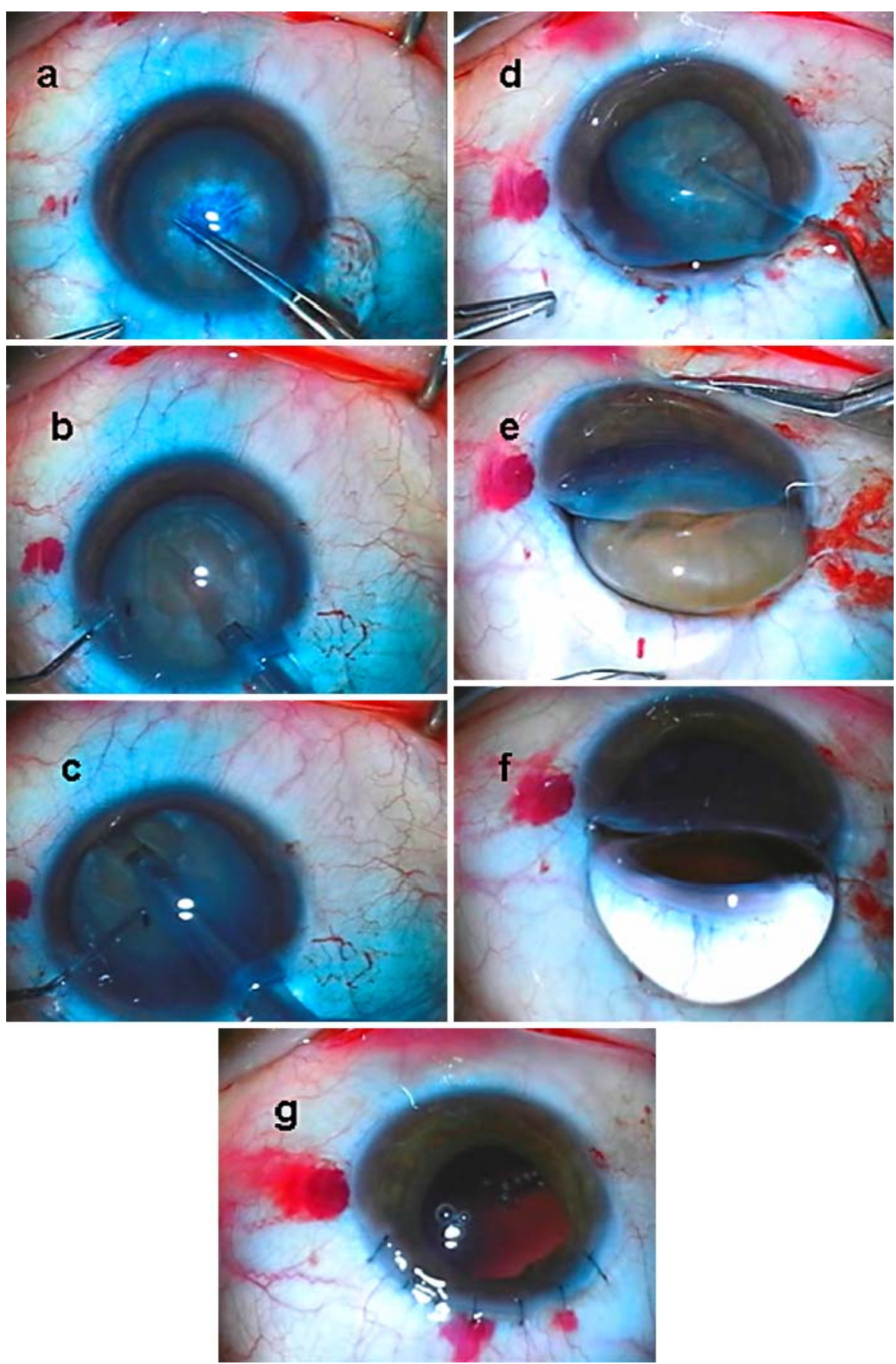

Fig. 2. a Completed capsulorhexis following anterior capsule staining. b Beginning of phacoemulsification - groove formation. c Lens subluxation during groove formation towards nasal inferior. d Significant zonular dehiscence is seen into nasal and superior quadrant approximately 180 degrees. e Expression of the lens. $\mathbf{f}$ Vitreous prolapsus with intact anterior vitreous face. $\mathbf{g}$ End of the operation after anterior vitrectomy and corneal suturation. 


\section{References}

1 Rowland LP, Shneider NA: Amyotrophic lateral sclerosis. N Engl J Med 2001;344:1688-1700.

-2 Muthialu A, Rauen M, Newsom TH, Jensen L, Oetting TA: Parachute-like harness to position patients with severe kyphosis during cataract surgery. J Cataract Refract Surg 2009;35:1332-1334.

3 Goetz CG: Amytrophic lateral sclerosis: early contributions of Jean-Martin Charcot. Muscle Nerve 2000;23:336-343.

4 Kokubo Y, Ito K, Kuzuhara S: Ophthalmomyiasis-like pigmentary retinopathy in ALS/PDC in the Kii peninsula of Japan. Neurology 2003;60:1725-1726.

-5 Kowalik MM, Smiatacz T, Pajuro R, Skowroński R, Trocha H, Nyka W, Raczyńska K, Wujtewicz M: Anaesthesia for ophthalmologic surgical procedures in a patient with advanced amyotrophic lateral sclerosis: a case report. Cases J 2008;1:338.

6 Baykal HE, Pleyer U, Sönnichsen K, Thiel HJ, Zierhut M: Severe eye involvement in pemphigus vulgaris. Ophthalmologe 1995;92:854-857.

7 Beele H, Claerhout I, Kestelyn P, Dierckxens L, Naeyaert JM, De Laey JJ: Bilateral corneal melting in a patient with paraneoplastic pemphigus. Dermatology 2001;202:147-150. 